\title{
(2) OPEN ACCESS \\ Incidence of necrotising enterocolitis before and after introducing routine prophylactic Lactobacillus and Bifidobacterium probiotics
}

\author{
Claire Robertson, ${ }^{1,2}$ George M Savva, ${ }^{3}$ Raducu Clapuci, ${ }^{1}$ Jacqueline Jones, ${ }^{1}$ \\ Hassan Maimouni, ${ }^{4}$ Eleanor Brown, ${ }^{1}$ Ashish Minocha, ${ }^{5}$ Lindsay J Hall, ${ }^{2}$ \\ Paul Clarke (D) ${ }^{1,4}$
}

\begin{abstract}
- Additional material is published online only. To view, please visit the journal online (http://dx.doi.org/10.1136/ archdischild-2019-317346)
\end{abstract}

${ }^{1}$ Neonatal Unit, Norfolk and Norwich University Hospitals NHS Foundation Trust, Norwich, UK

${ }^{2}$ Gut Microbes and Health, Quadram Institute Bioscience, Norwich, UK

${ }^{3}$ Core Science Resources

Quadram Institute Bioscience, Norwich, UK

${ }^{4}$ Norwich Medical School, University of East Anglia,

Norwich, UK

${ }^{5}$ Paediatric and Neonatal Surgery, Jenny Lind Children's Hospital, Norfolk and Norwich University Hospitals NHS

Foundation Trust, Norwich, UK

\section{Correspondence to}

Prof Paul Clarke, Neonatal Unit, Norfolk and Norwich University Hospitals NHS Foundation Trust, Norwich, UK:

paul.clarke@nnuh.nhs.uk

These data were previously presented in abstract form at the autumn meeting of the Neonatal Society, London, November 2018, and at the 7th Congress of the European Academy of Paediatric Societies (EAPS), Paris, France, November 2018.

Received 2 April 2019 Revised 3 August 2019 Accepted 24 September 2019 Published Online First 30 October 2019

\section{Check for updates}

(C) Author(s) (or their employer(s)) 2020. Re-use permitted under CC BY. Published by BMJ.

To cite: Robertson C, Savva GM, Clapuci R, et al. Arch Dis Child Fetal Neonatal Ed 2020:105:F380-F386.
ABSTRACT
Objective To compare rates of necrotising enterocolitis (NEC), late-onset sepsis, and mortality in 5-year epochs before and after implementation of routine daily multistrain probiotics administration in high-risk neonates.

Design Single-centre retrospective observational study over the 10-year period from 1 January 2008 to 31

December 2017.

Setting Level 3 neonatal intensive care unit (NICU) of the Norfolk and Norwich University Hospital, UK.

Patients Preterm neonates at high risk of NEC: admitted to NICU within 3 days of birth at $<32$ weeks' gestation or at 32-36 weeks' gestation and of birth weight $<1500 \mathrm{~g}$.

Intervention Prior to 1 January 2013 probiotics were not used. Thereafter, dual-species Lactobacillus acidophilus and Bifidobacterium bifidum combination probiotics were routinely administered daily to highrisk neonates; from April 2016 triple-species probiotics (L. acidophilus, B. bifidum, and B. longum subspecies infantis) were used.

Main outcome measures Incidence of NEC (modified Bell's stage 2a or greater), late-onset sepsis, and mortality.

Results Rates of NEC fell from 7.5\% (35/469 neonates) in the pre-implementation epoch to $3.1 \%$ (16/513 neonates) in the routine probiotics epoch (adjusted sub-hazard ratio $=0.44,95 \% \mathrm{Cl} 0.23$ to $0.85, \mathrm{p}=0.014$ ). The more than halving of NEC rates after probiotics introduction was independent of any measured covariates, including breast milk feeding rates. Cases of late-onset sepsis fell from 106/469 (22.6\%) to 59/513 $(11.5 \%)(p<0.0001)$, and there was no episode of sepsis due to Lactobacillus or Bifidobacterium. All-cause mortality also fell in the routine probiotics epoch, from $67 / 469(14.3 \%)$ to $47 / 513(9.2 \%)$, although this was not statistically significant after multivariable adjustment (adjusted sub-hazard ratio $=0.74,95 \% \mathrm{Cl} 0.49$ to 1.12 , $p=0.155)$.

Conclusions Administration of multispecies Lactobacillus and Bifidobacterium probiotics has been associated with a significantly decreased risk of NEC and late-onset sepsis in our neonatal unit, and no safety issues. Our data are consistent with routine use of Lactobacillus and Bifidobacterium combination probiotics having a beneficial effect on NEC prevention in very preterm neonates.
What is already known on this topic?

- Necrotising enterocolitis (NEC) remains a leading cause of mortality and morbidity in premature and very low birthweight infants.

- The aetiology of NEC is multifactorial, but the development of an abnormal gut microbiota is an important predisposing risk factor.

- Multiple meta-analyses and observational studies have shown that ameliorating gut bacterial colonisation through the enteral administration of live probiotic bacteria significantly reduces NEC incidence.

\section{What this study adds?}

- Routine daily dosing with Lactobacillus and Bifidobacterium probiotics was a cheap, simple intervention associated with significantly decreased NEC, surgical NEC and late-onset sepsis in our neonatal unit.

- No episode of sepsis due to Lactobacillus or Bifidobacterium occurred in the routine probiotic supplementation epoch.

- The apparent impact of reduced NEC incidence with probiotics was particularly pronounced in the first 2 weeks postnatal, implying that achieving early probiotic bacterial gut colonisation is crucial.

\section{INTRODUCTION}

Necrotising enterocolitis (NEC) is a leading cause of mortality and morbidity in premature very low birthweight (VLBW; <1500g) babies. ${ }^{12}$ Prevention of NEC is a top UK research priority. ${ }^{3}$ NEC aetiology is multifactorial, ${ }^{4}$ with abnormal gut microbiota increasingly recognised as central. ${ }^{5}$ Probiotics are 'live microorganisms that, when administered in adequate amounts, confer a health benefit on the host ${ }^{6}{ }^{6}$ Multiple randomised controlled trials (RCTs), systematic reviews and meta-analyses of RCTs and observational studies have shown that prophylactic probiotics prevent NEC in preterm babies. ${ }^{7-9}$ A 2012 meta-analysis, ${ }^{10}$ updated in 2017 to include 25 RCTs and $>7000$ neonates, ${ }^{11}$ showed strong evidence for using multispecies probiotics to reduce NEC incidence (pooled $\mathrm{OR}=0.36,95 \% \mathrm{CI}$ 
Table 1 NEC incidence and severity, sepsis incidence, and baseline data in the pre-probiotics vs routine probiotics epochs

\begin{tabular}{|c|c|c|}
\hline Factor & $\begin{array}{l}\text { Pre-probiotics epoch } \\
n=469\end{array}$ & $\begin{array}{l}\text { Routine probiotics } \\
\text { epoch } n=513\end{array}$ \\
\hline \multicolumn{3}{|l|}{ NEC diagnostic system } \\
\hline Modified Bell's $\geq 2 a$ & $35(7.5 \%)$ & $16(3.1 \%)$ \\
\hline Vermont-Oxford & $30(6.4 \%)$ & $15(2.9 \%)$ \\
\hline Battersby et al & $37(7.9 \%)$ & $19(3.7 \%)$ \\
\hline \multicolumn{3}{|l|}{ NEC severity } \\
\hline Modified Bell's 2a or 2b & $6(1.3 \%)$ & $2(0.4 \%)$ \\
\hline Modified Bell's 3a or 3b & $29(6.2 \%)$ & $14(2.7 \%)$ \\
\hline Surgical NEC & $30(6.4 \%)$ & $15(2.9 \%)$ \\
\hline Drains only & 4 & 1 \\
\hline Laparotomy \pm drain & 20 & 13 \\
\hline Died before laparotomy & 6 & 1 \\
\hline Postnatal age at NEC diagnosis (days) & $12(6-21)$ & $18(11-30)$ \\
\hline Postmenstrual age at NEC diagnosis (weeks) & $27(26-29)$ & $28(26-31)$ \\
\hline Mortality before discharge among NEC cases & $16(46 \%)$ & $3(19 \%)$ \\
\hline Spontaneous intestinal perforation & $2(0.4 \%)$ & $6(1.1 \%)$ \\
\hline \multicolumn{3}{|l|}{ Sepsis } \\
\hline Early onset sepsis & $10(1.1 \%)$ & $1(0.2 \%)$ \\
\hline Late-onset sepsis & $106(22.6 \%)$ & $59(11.5 \%)$ \\
\hline \multicolumn{3}{|l|}{ Responsible isolates* } \\
\hline CoNS & $87(18.6 \%)$ & $47(9.2 \%)$ \\
\hline Gram-negative & $19(4.1 \%)$ & $6(1.2 \%)$ \\
\hline Enterococcus & $11(2.3 \%)$ & $2(0.4 \%)$ \\
\hline Other organism & $15(3.2 \%)$ & $6(1.2 \%)$ \\
\hline Lactobacillus or Bifidobacterium sepsis & $0(0 \%)$ & $0(0 \%)$ \\
\hline Sex, female & $225(48 \%)$ & $261(51 \%)$ \\
\hline \multicolumn{3}{|l|}{ Mode of delivery } \\
\hline Vaginal & $193(42 \%)$ & $229(45 \%)$ \\
\hline Caesarean & $271(58 \%)$ & $284(55 \%)$ \\
\hline \multicolumn{3}{|l|}{ PROM } \\
\hline No & $306(65 \%)$ & $347(68 \%)$ \\
\hline Yes & $103(22 \%)$ & $118(23 \%)$ \\
\hline Not recorded & $60(13 \%)$ & $48(9 \%)$ \\
\hline \multicolumn{3}{|l|}{ NSAID treatment for PDA } \\
\hline None & $421(90 \%)$ & 487 (95\%) \\
\hline Indometacin & $37(8 \%)$ & $0(0 \%)$ \\
\hline Ibuprofen & $11(2 \%)$ & $26(5 \%)$ \\
\hline Birth weight (g) & $1100(810-1410)$ & $1100(832-1430)$ \\
\hline Gestational age (weeks) & $28(26-30)$ & $28(26-31)$ \\
\hline SGA & $114(24 \%)$ & $132(26 \%)$ \\
\hline Antenatal steroids & $405(87 \%)$ & $447(88 \%)$ \\
\hline Received initial antibioticst & $502 / 599(83.8 \%)$ & $509 / 597(85.6 \%)$ \\
\hline Days to first enteral feed & $2(1-4)$ & $2(2-3)$ \\
\hline \multicolumn{3}{|l|}{ Enteral feeding $\ddagger$} \\
\hline Never fed & $34(7 \%)$ & $32(6 \%)$ \\
\hline Mother's own breast milk only & $198(42 \%)$ & $322(63 \%)$ \\
\hline Donor breast milk only & $0(0 \%)$ & $9(2 \%)$ \\
\hline Preterm formula only & $50(11 \%)$ & $23(4 \%)$ \\
\hline Mixed (formula and any breast milk) & $186(40 \%)$ & $127(25 \%)$ \\
\hline
\end{tabular}

Data are median (IQR) or number (\%).

${ }^{*}$ For individual infants who had at least one such sepsis episode.

†Data presented are for all babies born at $<32$ weeks' gestation within the epochs who received initial empirical antibiotics (benzylpenicillin and gentamicin) following admission to NICU.

¥Mode of milk feeding between first feed and full feeds or NEC/death if earlier.

PDA, patent ductus arteriosus; CoNS, coagulase-negative staphylococci; NEC, necrotising enterocolitis; NICU, neonatal intensive care unit; NSAID, non-steroidal anti-inflammatory drug; PROM, prolonged rupture of membranes;SGA, small for gestational age.

0.24 to $0.53, \mathrm{p}<0.00001)$, and associated mortality $(\mathrm{OR}=0.58$, $95 \%$ CI 0.43 to $0.79, \mathrm{p}=0.0006)$. Yet at present most UK centres do not offer probiotics routinely. ${ }^{12}$ Uncertainties about effectiveness may arise from RCTs which have not shown benefit, heterogeneity, multiplicity, and dosage of probiotic microorganisms, differing inclusion criteria, treatment durations and NEC definitions used to assess benefit. ${ }^{13}$

There are no published reports on the safety or efficacy of routine probiotics used in any UK centres. We therefore aimed to assess whether routine probiotic supplementation has been associated with a decreased incidence of NEC, sepsis, and all-cause mortality in our centre.

\section{METHODS}

Single-centre retrospective review of neonates admitted to our tertiary-level neonatal intensive care unit (NICU) which caters to $\sim 6000$ deliveries annually and is a regional neonatal surgical centre.

\section{Eligible population}

'Eligible high-risk neonates' comprised all those inborn at $<32$ weeks' gestation, plus 32-36 weeks' gestation VLBW infants. Outborn babies were included if transferred in within 72 hours of birth, providing no abdominal concerns at referral.

\section{Study period}

Eligible neonates were reviewed in two consecutive 5-year epochs, allocated by date of birth: 1 January 2008 to 31 December 2012 (pre-probiotics epoch) and 1 January 2013 to 31 December 2017 (routine probiotic epoch).

\section{Enteral feeding practices}

Our unit adopted a standardised regional enteral feeding guideline for preterm neonates in January 2011. Ideally, human milk feeds commenced on day 1. From May 2013, donor breast milk (DBM) was available to supplement shortfalls in mother's own breast milk supply before full feeds. Cow's milk-based fortifier was added to breast milk between full enteral feeds $(\geq 150 \mathrm{~mL} /$ $\mathrm{kg}$ /day) and discharge-a consistent policy across epochs. Milk type used in the period until full feeds was categorised as exclusive maternal breast milk, exclusive DBM, formula, or mixed (ie, any combination of own maternal milk, DBM, and formula).

\section{Probiotic exposure and compliance}

We introduced routine daily combination Lactobacillus and Bifidobacterium probiotics for prophylaxis of NEC in high-risk babies resident in our NICU on 1 January 2013, and for all eligible infants subsequently admitted. ${ }^{14}$ Written parental information was provided. ${ }^{15}$ We administered first probiotic dose with first colostrum feeds, ideally on postnatal day 1. Probiotics continued until $\sim 34$ weeks postmenstrual age. We initially used the dual-species probiotic Infloran capsules (Desma Healthcare, Chiasso, Switzerland), containing Lactobacillus acidophilus and Bifidobacterium bifidum, half a capsule $(125 \mathrm{mg})$ twice daily $\left(1 \times 10^{9}\right.$ colony-forming units (CFU) of each bacterial species daily). From April 2016, we used triple-species Labinic Drops (Biofloratech, Walton-on-Thames, UK), four drops once daily $\left(\sim 0.5 \times 10^{9}\right.$ CFU dosage each of L. acidophilus, B. bifidum and B. longum subspecies infantis daily). Probiotic batches were subject to quality control (LJH's laboratory) for species and CFU confirmation alongside our prospective microbiota study. ${ }^{16}$ Probiotics were prescribed on drug charts in accordance with our NICU's guideline, and compliance was good. ${ }^{17}$

\section{Oxygen saturation limits}

Between 2008 and May 2011, we targeted oxygen saturation $\left(\mathrm{SaO}_{2}\right)$ range $84 \%-92 \%$ for babies aged $<32$ weeks. From 31 
May 2011, with emergent evidence associating lower $\mathrm{SaO}_{2}$ ranges with mortality and $\mathrm{NEC},{ }^{18}$ we targeted $91 \%-95 \% \mathrm{SaO}_{2}$.

\section{Identification and classification of NEC cases}

From BadgerNet electronic patient records (CleverMed, UK), we identified all cases of definite, suspected, and perforated NEC. All eligible infants were screened between admission and final discharge home (or death if earlier) from either our NICU/paediatric ward, or step-down NICU/other hospital ward if transferred out. Electronic clinical records, including X-ray request forms, abdominal radiographs, radiologist reports, blood results, surgical notes, histopathology and autopsy reports, and death certificates were independently reviewed by two unblinded clinicians (CR, PC) to determine whether they met NEC case definition according to each of three diagnostic systems: modified Bell's staging criteria, ${ }^{19}$ Vermont-Oxford Network, ${ }^{20}$ and Battersby et al. ${ }^{1}$ We defined 'surgical NEC' as any baby with definite NEC in whom an abdominal drain was inserted or who underwent laparotomy. Potential surgical NEC cases whose operative findings and/or histopathology reports were uncertain or required expert interpretation, and also those with uncertainty regarding staging, were additionally reviewed and adjudicated by a consultant paediatric surgeon (AM) blinded to epoch.

We imputed as either NEC Bell's stage $2 \mathrm{~b} / 3 \mathrm{a}$ cases babies who had acute clinical deterioration with Bell's stage 2 or 3 systemic and gastrointestinal signs (eg, blood in stool, abdominal distension, tenderness, acidosis), but without classic stage
2/3 radiographic findings of pneumatosis intestinalis, portal venous gas or pneumoperitoneum, provided treated clinically as presumed NEC with $\geq 10$ days' antibiotics and nil enterally and with fixed distended loops, definite ascites or gasless abdomen on abdominal X-ray and no alternative diagnosis (eg, sepsis) for the episode. These included babies who underwent surgical drain placement who died before planned laparotomy without autopsy, but with NEC recorded on their death certificate.

Isolated pneumoperitoneum without other clinical/radiographic evidence of NEC was diagnosed as spontaneous intestinal perforation, not NEC. Similarly, any case who satisfied any staging/clinical-radiological definition of NEC but who at proximate surgery or postmortem examination for that episode had no NEC findings was excluded as NEC.

\section{Identification and classification of late-onset sepsis}

Late-onset sepsis was defined as clinical signs of sepsis and a concomitant positive blood or cerebrospinal fluid culture occurring $>72$ hours after birth and before 46 weeks' postmenstrual age or discharge/transfer from our NICU if sooner. We reviewed all positive blood and CSF cultures from our local microbiology laboratory database for the whole study period. Repeated growth of the same isolate in successive samples obtained within 7 days was considered the same episode.

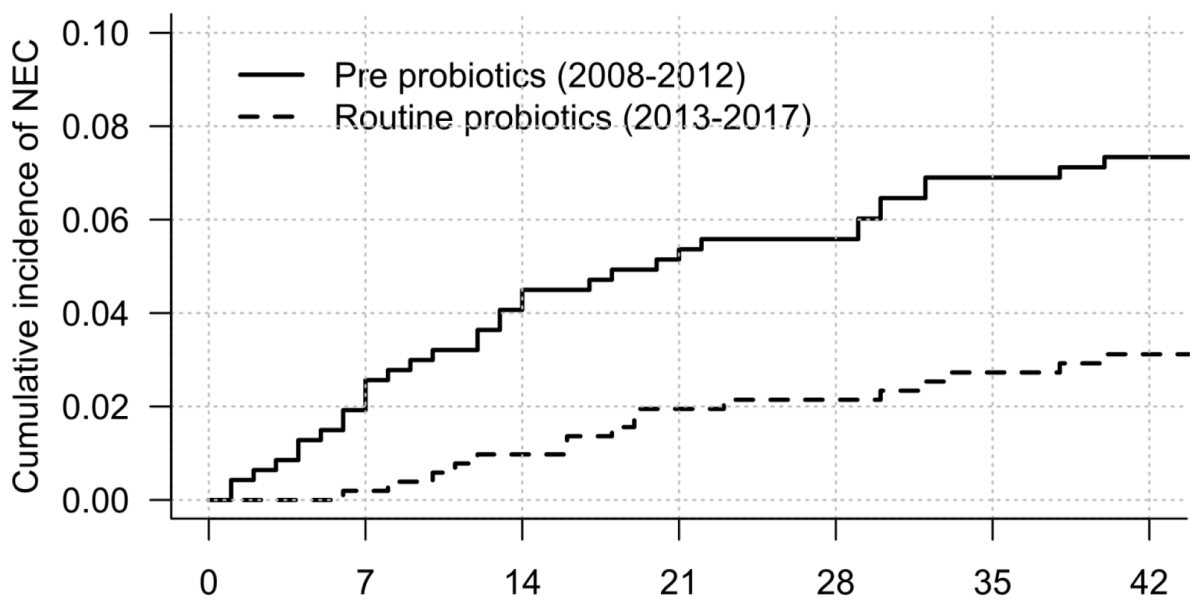

Pre probiotics

Days since birth

$\begin{array}{rccccccc}\text { At risk } & 469 & 431 & 410 & 375 & 332 & 277 & 227 \\ \text { Discharged home } & 0 & 0 & 2 & 21 & 53 & 101 & 147 \\ \text { Died without NEC } & 0 & 27 & 35 & 41 & 47 & 47 & 49 \\ \text { NEC } & 0 & 9 & 19 & 24 & 26 & 32 & 34 \\ \text { Routine probiotics } & & & & & & & \\ \text { At risk } & 513 & 483 & 472 & 448 & 400 & 344 & 293 \\ \text { Discharged home } & 0 & 0 & 3 & 21 & 67 & 118 & 165 \\ \text { Died without NEC } & 0 & 29 & 33 & 34 & 35 & 37 & 39 \\ \text { NEC } & 0 & 1 & 5 & 10 & 11 & 14 & 16\end{array}$

Figure 1 The cumulative incidence of necrotising enterocolitis (NEC) from date of birth stratified by epoch. Risk table shows the number of cases still at risk with each passing week from birth, as well as the cumulative numbers of those diagnosed with NEC, who died without a diagnosis of NEC, or who were discharged home without a diagnosis of NEC. One NEC case was censored in this time-to-event analysis, a baby born in the month before 1 January 2013 who developed NEC after that date, leaving 34 cases in the pre-probiotics epoch and 16 cases in the routine probiotics epoch included. 


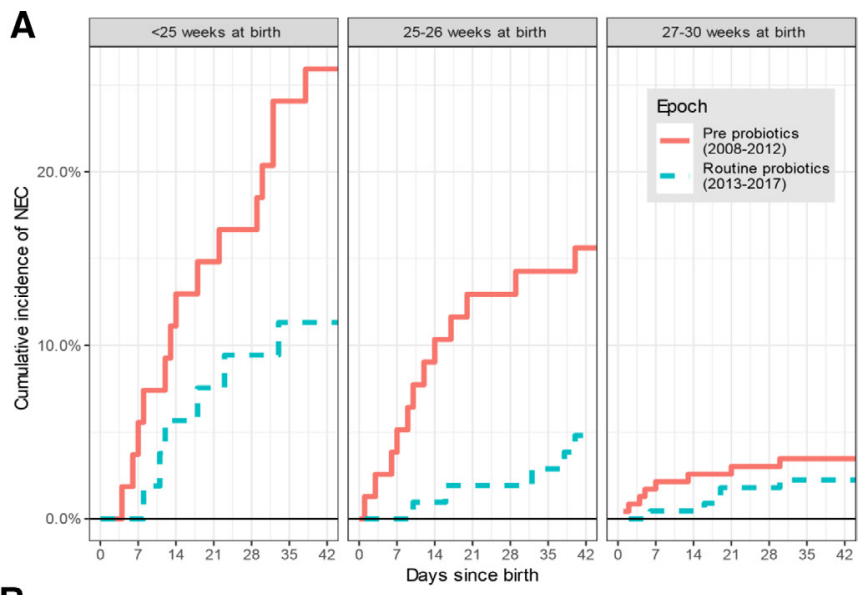

B
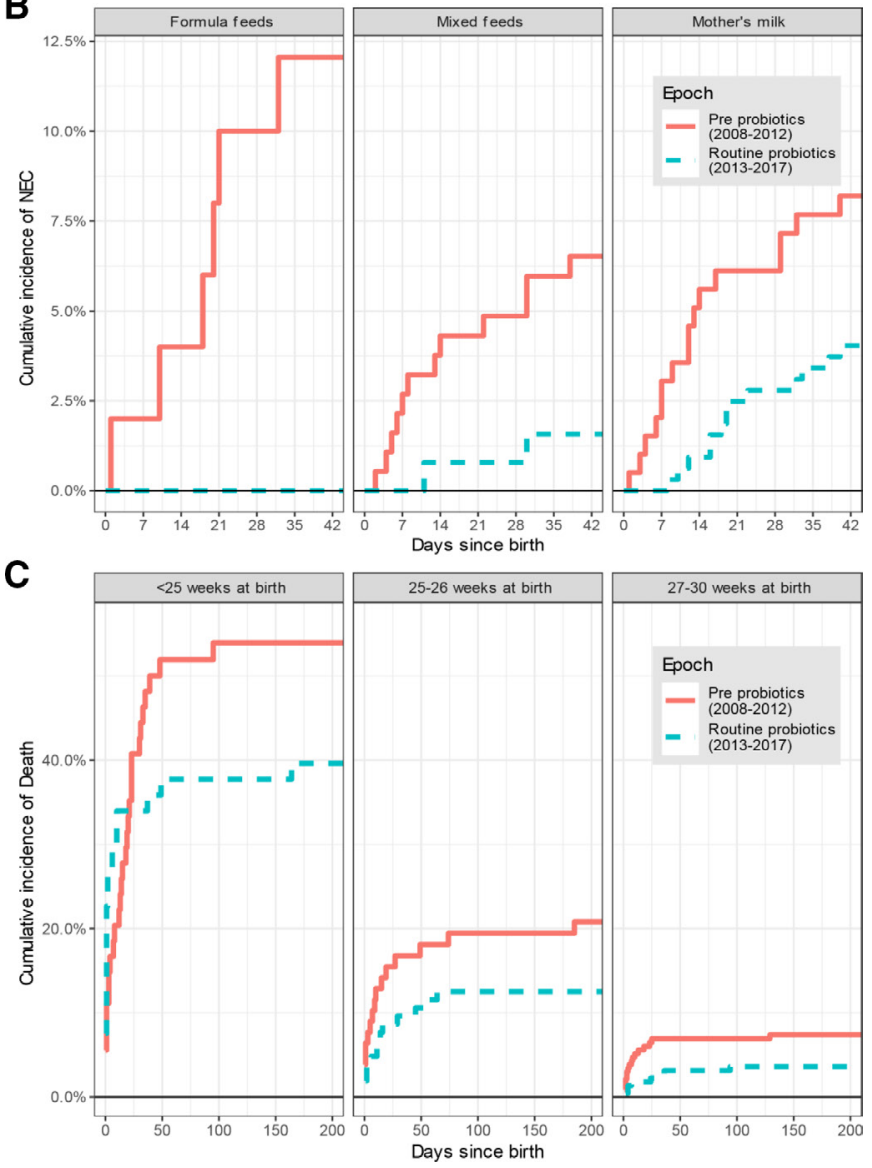

Figure 2 (A) Cumulative incidence of necrotising enterocolitis (NEC) stratified by gestational age at birth and by epoch. (B) Cumulative incidence of NEC stratified by gestational age at birth and by milk type for those who initiated enteral feeds. There was no case of NEC among those fed donor breast milk exclusively and so this group is not shown. (C) Cumulative incidence of mortality up to 200 days after birth, stratified by epoch and gestational age.

\section{Outcome measures}

NEC was coded as a binary outcome, and NEC case definition used for primary analyses was modified Bell's criteria $\geq 2 \mathrm{a}$. ${ }^{19}$ Mortality from any cause was included as a secondary outcome.

\section{Statistical analysis}

Full details of statistical methods are provided in online supplementary figure S1. In short, time-to-event analysis was used to estimate the difference in NEC rate between epochs, controlling for potentially confounding variables, and with death and discharge considered competing risks. Cumulative NEC incidence is reported, with sub-hazard ratios (sub-HR: ratio of cumulative incidence rates) reflecting the association between each factor and cumulative incidence.

Effect of epoch appeared to vary significantly over the risk period, thus we conducted a second analysis including three separate coefficients for the effect of epoch on NEC in the first, second and beyond the second week after birth. A regression discontinuity analysis ${ }^{21}$ assessed whether the effect of epoch would be better explained by a linear trend over time or by a step change coinciding with introduction of probiotics. A time-toevent analysis estimated the effect of epoch on all-cause mortality. We used $\chi^{2}$ and Fisher's exact tests to compare mortality rates following NEC diagnosis and other between-epoch proportions.

\section{RESULTS}

\section{Descriptive analysis}

The sample included 982 eligible neonates: 469 born in the pre-probiotics epoch and 513 in the routine probiotics epoch (online supplementary figure S2, patient flow). Table 1 shows comparative distributions of covariates between epochs. The only covariate to differ substantially between epochs was mode of milk feeding in the period up to full feeds: exclusive breast

Table 2 Multivariable regression showing the relative risk of NEC associated with each factor

\begin{tabular}{|c|c|c|c|}
\hline Factor & Level & Sub-HR $(95 \% \mathrm{Cl})$ & $P$ value \\
\hline Epoch & $\begin{array}{l}\text { Routine vs pre- } \\
\text { probiotics }\end{array}$ & $0.44^{*}(0.23$ to 0.85$)$ & 0.014 \\
\hline \multirow{4}{*}{$\begin{array}{l}\text { Gestational age } \\
\text { at birth }\end{array}$} & $<25$ weeks & 1.00 (ref) & \\
\hline & 25-26 weeks & $0.34^{* *}$ (0.16 to 0.70$)$ & 0.003 \\
\hline & 27-30 weeks & $0.09^{* * *}(0.03$ to 0.26$)$ & $<0.001$ \\
\hline & $\geq 31$ weeks & - & - \\
\hline \multirow[t]{5}{*}{ Milk type† } & Mother's milk & 1.00 (ref) & \\
\hline & No enteral feed & $0.15^{* *}$ (0.04 to 0.52$)$ & 0.003 \\
\hline & Donor milk & - & - \\
\hline & Formula feed & 0.85 (0.29 to 2.52 ) & 0.775 \\
\hline & Mixed & 0.82 (0.41 to 1.64$)$ & 0.576 \\
\hline \multirow[t]{3}{*}{ Birth weight } & $<1000 \mathrm{~g}$ & 1.00 (ref) & \\
\hline & $1000-1499 \mathrm{~g}$ & 0.71 (0.26 to 1.99 ) & 0.520 \\
\hline & $\geq 1500 \mathrm{~g}$ & $1.12(0.22$ to 5.84$)$ & 0.891 \\
\hline Sex & Male (vs female) & 1.05 (0.59 to 1.86$)$ & 0.872 \\
\hline Antenatal steroids & $\begin{array}{l}\text { At least one dose (vs } \\
\text { never) }\end{array}$ & 1.10 (0.45 to 2.68$)$ & 0.840 \\
\hline \multirow[t]{3}{*}{ NSAID } & None & 1.00 (ref) & \\
\hline & Indometacin & 1.21 (0.47 to 3.08 ) & 0.694 \\
\hline & Ibuprofen & 1.20 (0.41 to 3.56$)$ & 0.738 \\
\hline \multirow[t]{3}{*}{ PROM } & No & 1.00 (ref) & \\
\hline & Yes & 0.53 (0.22 to 1.29 ) & 0.164 \\
\hline & Not recorded & 0.78 (0.33 to 1.88$)$ & 0.583 \\
\hline Mode of birth & $\begin{array}{l}\text { Caesarean (vs } \\
\text { vaginal) }\end{array}$ & 1.82 (0.92 to 3.60$)$ & 0.086 \\
\hline
\end{tabular}

There were no cases of NEC among those fed using donor milk, or those born after 31 weeks in either epoch and so these groups are excluded from regression analysis.

${ }^{*} \mathrm{P}<0.05,{ }^{* *} \mathrm{p}<0.01,{ }^{* *} \mathrm{p}<0.001$

tMode of milk feeding between first feed and full feeds or NEC/death if earlier. NEC, necrotising enterocolitis; NSAID, non-steroidal anti-inflammatory drug; PROM, premature rupture of membranes; Ref., reference group; Sub-HR, sub-hazard ratio. 


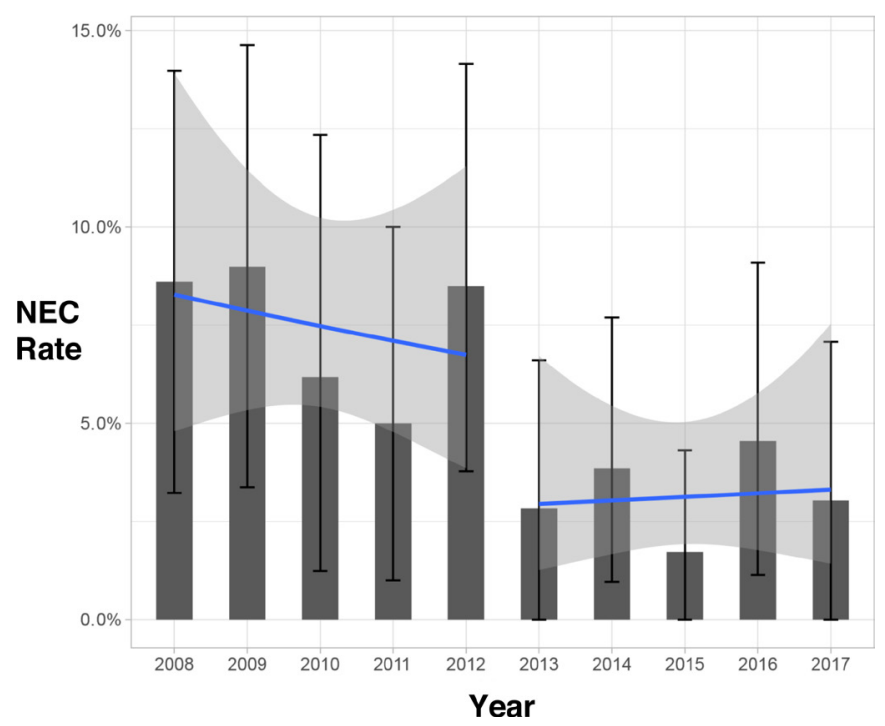

Figure 3 The rate of necrotising enterocolitis (NEC) in each year (solid bars with error bars), and linear trends estimated within each era (lines with error bands). Each estimate is shown with a $95 \%$ bootstrapped confidence interval.

milk feeding was more common in the routine probiotics epoch (table 1).

\section{NEC incidence according to diagnostic system used}

Table 1 shows NEC incidence in the pre-probiotics and routine probiotics epochs using the three NEC classifications. NEC Bell's stage $\geq 2$ a occurred in $35(7.5 \%)$ babies in the preprobiotics epoch and $16(3.1 \%)$ in the routine probiotics epoch. Imputed cases (without classical X-ray signs) were $n=5$ in the pre-probiotics epoch and $n=1$ in the routine probiotics epoch. In the latter era, three babies who developed NEC during the second postnatal week had not received prior probiotics; dosing had been overlooked in two cases. NEC rates within the routine probiotics epoch were similar irrespective of product used (10 cases during 39 months using Infloran; 6 cases during 21 months using Labinic). More NEC cases of Bell's stage $\geq 3$ a occurred in the pre-probiotics era, $29(6.2 \%)$ vs $14(2.7 \%)(p=0.017)$, but comprised a similar proportion of overall NEC cases within epochs, $29 / 35(83 \%)$ vs $14 / 16(88 \%)(p=0.70)$. Surgical NEC occurred in 30 infants in the pre-probiotics epoch $(6.4 \%$ of eligible neonates) vs $15(2.9 \%)$ in the routine probiotics epoch $(p=0.014)$.

\section{Cumulative incidence of NEC from birth}

Figure 1 shows the cumulative incidence of NEC from day of birth until final discharge home (or earlier death). NEC incidence was strongly associated with birth gestational age (GA) (figure 2). Irrespective of GA, all cases of NEC occurred within 40 days of birth. NEC rate was lower in the routine probiotic-use era for all GA subgroups, with differences particularly pronounced in the first 2 weeks after birth.

The overall sub-HR for being born in the routine probiotics compared with pre-probiotics epoch on NEC rate was 0.43 (95\% CI 0.23 to $0.77, p=0.005)$. When all other covariates were included in the model (table 2), the estimated association between epoch and NEC rate remained very similar (sub-HR $=0.44$, $95 \%$ CI 0.23 to $0.85, \mathrm{p}=0.014)$. However, as reflected in the cumulative incidence curve, the difference between epochs was greatest in the first week after birth, and there was no difference after the second week (test for proportional hazard vs difference in sub-HR over time since birth: $p=0.013)$. Estimates for the association between NEC and probiotics stratified by time since birth are shown in online supplementary table S3. In the first week after birth, the sub-HR for routine versus no probiotics was $0.08(95 \%$ CI 0.01 to $0.59, p=0.014)$, in the second week was $0.43(95 \%$ CI 0.13 to $1.4, \mathrm{p}=0.17)$ and thereafter was 0.85 (95\% CI 0.36 to $2.0, \mathrm{p}=0.72$ ).

NEC was also independently linked to GA, and appeared less common before initiation of enteral feeds. There was no evidence that birth weight (once adjusted for GA) or milk type used for feeding were significantly associated with NEC rates, although our study was not designed to test these associations.

\section{All-cause mortality and NEC-associated rate of death within epochs}

There were 67 deaths from all causes before discharge in the pre-probiotics epoch (14.3\% of eligible neonates) and 47 in the routine probiotics epoch $(9.2 \%)$. The cumulative incidence of death among eligible included babies is shown in figure 2, stratified by epoch and GA. All-cause mortality reduced between epochs (sub-HR $=0.62,95 \% \mathrm{CI} 0.42$ to $0.90, \mathrm{p}=0.013$ ). This was attenuated and no longer statistically significant after adjusting for all covariates (online supplementary table S4). However, as with NEC, the proportional hazard assumption was not met and on stratifying by time since birth there was a significantly lower death rate beyond 2 weeks during the routine probiotics epoch compared with in the pre-probiotics epoch (sub-HR $=0.34,95 \%$ CI 0.17 to $0.68, \mathrm{p}=0.003$; online supplementary table S5). Other factors independently associated with mortality were lower GA, never having received any enteral milk feeds, and non-reception of antenatal steroids.

Death before discharge among diagnosed NEC cases occurred in $15 / 35$ (44\%) in the pre-probiotics epoch, compared with $3 / 16$ $(19 \%)$ in the routine probiotics epoch, $\mathrm{p}=0.15$.

\section{Regression discontinuity analysis}

Within individual years across the whole study period, the proportion of NEC cases as a proportion of all eligible admissions is shown in figure 3, along with estimates of the trend across years within each epoch. After adjusting for calendar year as a continuous variable in the time-to-event analysis, the effect of epoch was largely unchanged and remained statistically significant in the first week after birth (online supplementary table S3). This suggests that our results are not explained by an underlying linear improvement in NEC rates with time, but rather reflect a step change in incidence around the introduction of routine probiotics administration. Conversely, the improvement in death rate between epochs is better explained by a linear trend over time (online supplementary table S5).

\section{Sensitivity analyses}

Estimates were similar when postmenstrual age was used as a time-scale instead of days since birth (data not shown). Since milk type used between first and full feeds was the only other recorded variable to change significantly between epochs, we also stratified plots by mode of milk feed (among those who initiated enteral feeds) (figure 2); this showed a clear reduction in NEC rate in the routine probiotics epoch irrespective of milk feed type. Replacing 'birth weight' with 'small for gestational age' in the multivariable analysis did not affect findings. Repeating analyses using the Vermont-Oxford Network NEC case definition ${ }^{20}$ did not significantly affect findings either: the estimate of effect 
of epoch was sub-HR=0.47 (95\% CI 0.24 to $0.92, p=0.027$ ), and the stratified analysis was also largely consistent. The only difference was in the regression discontinuity analysis, where the evidence was less strong that the trend was due to probiotics introduction as opposed to a linear trend (in the first week after birth, adjusted sub-HR $=0.09,95 \%$ CI 0.00 to $1.62, p=0.10$ ), although this involved a very small number and was still consistent with a big drop in NEC rate in the first week after birth.

\section{Incidence of late-onset sepsis}

Babies having one or more episodes of late-onset sepsis were significantly fewer in the routine probiotics epoch compared with the previous epoch $(p<0.0001)$, and there were fewer episodes of coagulase-negative staphylococcal sepsis $(\mathrm{p}<0.0001)$ (table 1).

No case of any probiotic-organism bacterial sepsis was observed in either epoch.

\section{DISCUSSION}

\section{Summary of findings}

In this sample of 982 high-risk neonates admitted to our NICU over a 10-year period, NEC rate fell by more than half (from $7.5 \%$ to $3.1 \%$ ) in the 5 -year period following the introduction of a policy of routine multispecies probiotics administration from birth ( $\sim 55 \%$ relative risk reduction). Our apparent more-than halving of NEC risk with probiotics mirrors remarkably the highly significant risk reductions shown in large meta-analyses of RCTs and observational studies, ${ }^{7-11}$ including that of a very large German observational study which also used Infloran. ${ }^{22}$ While some quality improvements occurred in our NICU over the study period, the difference observed was not attributable to changes in the main important covariates assessed between epochs, including exposure to breast milk. The improvement affected all high-risk neonates irrespective of GA. The impact appeared greatest in the first 2 weeks after birth, suggesting that aiming for very early postnatal probiotic bacterial gut colonisation is crucial. Late-onset sepsis rates also halved in the routine probiotics epoch compared with in the previous epoch (from $22.6 \%$ to $11.5 \%)$. There was a fall in the all-cause mortality rate between epochs, although this was more consistent with a continuous trend over the study period.

\section{Strengths and limitations}

This is the first UK study to evaluate the potential impact of routine probiotics use on NEC rates. We used multispecies Lactobacillus and Bifidobacterium probiotics, shown to be most effective for NEC prophylaxis in large meta-analysis. ${ }^{11}$ These were routinely available, and commercially produced to the same accredited Good Manufacturing Practice standards required for drug production. We had a high rate of compliance and early probiotics administration in eligible babies. We applied three different NEC classification systems; all showed similar reductions in NEC rates across epochs. A further study strength is the variety of rigorous statistical analyses applied.

Being retrospective and observational, our findings only support an association and cannot conclusively determine that probiotics caused the observed markedly reduced NEC incidence; unmeasured confounders might underlie observed differences between epochs. The only measured covariate observed to change between epochs was milk-type used, but this did not explain the improved NEC rate. Furthermore, a regression discontinuity analysis suggested that the drop in NEC rate was better explained by a step-change at the introduction of probiotics, and no other significant policy changes occurred between epochs that could have affected NEC incidence so markedly.

Importantly, colonisation and development of the early life microbiota, including Bifidobacterium and Lactobacillus species, occurs immediately postbirth. Previous studies (clinical and preclinical) indicate that optimal colonisation by supplemented species in a 'naïve' gut environment enables more effective persistence, linking to improved short-term and long-term host health..$^{23-25}$

Choice of bacterial species and strains is a crucial consideration. Many previous studies already highlighted that strains of species B. bifidum, B. longum subspecies infantis, and $L$. acidophilus were efficacious in other NICUs globally, key to our selecting these combinations for routine use.

Mechanistically, B. bifidum and B. infantis strains are pioneer colonisers of the infant gut, and their innate ability to digest components of breast milk preferentially, for example, human milk oligosaccharides (HMOs), augments their establishment within the infant gut. ${ }^{26}{ }^{27}$ Numerous studies have shown how these species may provide resistance to potentially pathogenic microbes, so-called 'colonisation resistance', and also aid development of the mucosal and systemic immune systems, key beneficial traits to enhance development of the preterm gut and prevent NEC and late-onset sepsis. ${ }^{25} 28$ Furthermore, a multispecies approach may promote better colonisation because Lactobacillus may serve to reduce the oxygen content in the neonatal gastrointestinal tract, facilitating colonisation and persistence of anaerobic (ie, oxygen sensitive) Bifidobacterium species. ${ }^{24}$ However, it is critical to note that there are significant differences between individual Bifidobacterium and Lactobacillus species and also huge strain variability, not least in ability to digest dietary components such as HMOs and in their immune and infection modulatory traits. Such complexities may explain conflicting results of some previous RCTs, such as the large UK multicentre Probiotics in Preterm infants Study (the PiPS trial) which-using the single-strain probiotic B. breve BBG-001—did not reduce NEC or late-onset sepsis. ${ }^{29}$ Those findings may link to poor colonisation ability of the selected B. breve BBG-001 strain, ${ }^{30}$ compounded by low dosage used, ${ }^{31}$ and inability of this strain to digest key early life dietary components. $B$. breve strains frequently lack the genes required to assimilate $\mathrm{HMO}$ molecules, and consequently have limited ability to assimilate HMOs compared with $B$. infantis and B. bifidum. ${ }^{32}$ Further studies are needed to determine the generalisability of the underlying potential benefits of multispecies probiotics in this at-risk cohort.

\section{CONCLUSION}

Administration of multispecies Lactobacillus and Bifidobacterium combination probiotics has been associated with a significantly decreased risk of NEC and late-onset sepsis in our NICU. This evaluation supports our routine use of multi-species Lactobacillus and Bifidobacterium combination probiotics for preventing NEC in very preterm neonates.

\section{Twitter Paul Clarke@drpaulclarke}

Acknowledgements The authors would like to thank the following colleagues for their support of the initiative: Dr Mary-Anne Morris and the Jenny Lind Nutritional Support Team, all neonatal nursing, medical, and surgical colleagues, Dr Catherine Tremlett (microbiologist), Colin Green (pharmacist), Dr Tarnya Marshall (Chair of the Trust's Drug, Therapeutics \& Medicines Management Committee) and Julie Dawson ( $R+D$ Services Manager). The authors would also like to thank the anonymous reviewers for helpful comments and suggestions on the earlier manuscript version. 
Contributors PC devised the study. CR, JJ, RC, HM, EB and PC collected the data. CR, PC and AM adjudicated NEC cases. GMS provided statistical expertise and analysed the data. CR, GMS, LJH and PC wrote the first manuscript draft and PC wrote the final draft. LJH provided intellectual input. All authors contributed to manuscript revisions and approved the final version. PC is guarantor.

Funding This work was part funded by: a Wellcome Trust Investigator award to LJH (100974/C/13/Z); a BBSRC ISP grant for Gut Health and Food Safety to LJH (BB/ J004529/1); BBSRC ISP grants for Gut Microbes and Health BB/R012490/1 and its constituent project(s) (BBS/E/F/000PR10353 and BBS/E/F/000PR10355) to LJH. GMS was supported by the BBSRC Core Capability Grant BB/CCG1860/1. CR received a Bliss travel bursary sponsored by Chiesi, which supported conference presentation of data from this study.

Competing interests None declared.

Patient consent for publication Not required.

Ethics approval This project was a registered clinical audit/service evaluation. It was reviewed by the R+D Services Manager of the Norfolk and Norwich University Hospitals NHS Foundation Trust and was considered exempt from requiring formal research ethics service approval.

Provenance and peer review Not commissioned; externally peer reviewed. Data availability statement Data are available on reasonable request.

Open access This is an open access article distributed in accordance with the Creative Commons Attribution 4.0 Unported (CC BY 4.0) license, which permits others to copy, redistribute, remix, transform and build upon this work for any purpose, provided the original work is properly cited, a link to the licence is given, and indication of whether changes were made. See: https://creativecommons.org/ licenses/by/4.0/.

ORCID iD

Paul Clarke http://orcid.org/0000-0001-6203-7632

\section{REFERENCES}

1 Battersby C, Longford N, Costeloe K, et al. Development of a gestational agespecific case definition for neonatal necrotizing enterocolitis. JAMA Pediatr 2017:171:256-63.

2 Neu J, Pammi M. Pathogenesis of NEC: impact of an altered intestinal microbiome. Semin Perinatol 2017;41:29-35.

3 Duley L, Uhm S, Oliver S, et al. Top 15 UK research priorities for preterm birth. The Lancet 2014;383:2041-2.

4 Gordon PV, Swanson JR, MacQueen BC, et al. A critical question for NEC researchers: can we create a consensus definition of NEC that facilitates research progress? Semin Perinatol 2017:41:7-14.

5 Berrington JE, Stewart CJ, Embleton ND, et al. Gut microbiota in preterm infants: assessment and relevance to health and disease. Arch Dis Child Fetal Neonatal Ed 2013;98:F286-90

6 Hill C, Guarner F, Reid G, et al. The International scientific association for probiotics and prebiotics consensus statement on the scope and appropriate use of the term probiotic. Nat Rev Gastroenterol Hepatol 2014;11:506-14.

7 AlFaleh $\mathrm{K}$, Anabrees J. Probiotics for prevention of necrotizing enterocolitis in preterm infants. Cochrane Database Syst Rev 2014:CD005496.

8 Olsen R, Greisen G, Schrøder M, et al. Prophylactic probiotics for preterm infants: a systematic review and meta-analysis of observational studies. Neonatology 2016;109:105-12.

9 Dermyshi E, Wang Y, Yan C, et al. The "Golden Age" of Probiotics: A Systematic Review and Meta-Analysis of Randomized and Observational Studies in Preterm Infants. Neonatology 2017;112:9-23

10 Wang Q, Dong J, Zhu Y. Probiotic supplement reduces risk of necrotizing enterocolitis and mortality in preterm very low-birth-weight infants: an updated meta-analysis of 20 randomized, controlled trials. J Pediatr Surg 2012;47:241-8.
11 Chang $\mathrm{H}-\mathrm{Y}$, Chen J-H, Chang J-H, et al. Multiple strains probiotics appear to be the most effective probiotics in the prevention of necrotizing enterocolitis and mortality: an updated meta-analysis. PLoS One 2017;12:e0171579.

12 Duffield SD, Clarke P. Current use of probiotics to prevent necrotising enterocolitis. Arch Dis Child Fetal Neonatal Ed 2019;104:F228

13 van den Akker CHP, van Goudoever JB, Szajewska H, et al. Probiotics for preterm infants: a strain-specific systematic review and network meta-analysis. J Pediatr Gastroenterol Nutr 2018;67:103-22.

14 Deshpande GC, Rao SC, Keil AD, et al. Evidence-Based guidelines for use of probiotics in preterm neonates. BMC Med 2011:9:92.

15 Sesham R, Oddie S, Embleton ND, et al. Probiotics for preterm neonates: parents' perspectives and present prevalence. Arch Dis Child Fetal Neonatal Ed 2014;99:F345-345.

16 Alcon-Giner C, Dalby M, Caim S, et al. Microbiota supplementation with Bifidobacterium and Lactobacillus modifies the preterm infant gut microbiota and metabolome. bioRxiv 2019;698092.

17 Brown E, Dabbour S, Clapuci R, et al. 4 years' routine administration of probiotics to high-risk neonates: retrospective audit of adherence ot local guidelines. 7th Congress of the European Academy of Paediatrc Societies (EAPS) October 30-November 3,2018; Paris, France. Available: https://public-poster-links.s3.amazonaws.com/EAPS2018/EAPS-2018-EAPS8-0834.pdf [Accessed 18 Oct 2019].

18 Stenson B, Brocklehurst P, Tarnow-Mordi W, et al. Increased 36-week survival with high oxygen saturation target in extremely preterm infants. $N$ Engl I Med 2011;364:1680-2.

19 Walsh MC, Kliegman RM. Necrotizing enterocolitis: treatment based on staging criteria. Pediatr Clin North Am 1986;33:179-201.

20 Vermont Oxford Network. 2019 Manual of Operations, Part 2: Data Definitions \& Infant Data Forms. Release 23.2, February 2019. Available: https://vtoxford.zendesk. com/hc/en-us/article_attachments/360024732954/Manual_of_Operations_Part_2_ V23.2.pdf [Accessed 18 Oct 2019].

21 Thistlethwaite DL, Campbell DT. Regression-discontinuity analysis: an alternative to the ex post FACTo experiment. J Educ Psychol 1960;51:309-17.

22 Denkel LA, Schwab F, Garten L, et al. Protective effect of Dual-Strain probiotics in preterm infants: a multi-center time series analysis. PLoS One 2016:11:e0158136.

23 Houghteling PD, Walker WA. Why is initial bacterial colonization of the intestine important to Infants' and Children's health? J Pediatr Gastroenterol Nutr 2015;60:294-307.

24 Koskella B, Hall LJ, Metcalf CJE. The microbiome beyond the horizon of ecological and evolutionary theory. Nat Ecol Evol 2017;1:1606-15.

25 O'Neill I, Schofield Z, Hall LJ. Exploring the role of the microbiota member Bifidobacterium in modulating immune-linked diseases. Emerging Topics in Life Sciences 2017;1:333-49.

26 Frese SA, Hutton AA, Contreras LN, et al. Persistence of Supplemented Bifidobacterium longum subsp. infantis EVCO01 in Breastfed Infants. mSphere 2017:2:501-17.

27 O'Callaghan A, van Sinderen D. Bifidobacteria and their role as members of the human gut microbiota. Front Microbiol 2016;7:925.

28 Fukuda S, Toh H, Taylor TD, et al. Acetate-producing bifidobacteria protect the host from enteropathogenic infection via carbohydrate transporters. Gut Microbes 2012;3:449-54.

29 Costeloe K, Hardy P, Juszczak E, et al. Bifidobacterium breve BBG-001 in very preterm infants: a randomised controlled phase 3 trial. The Lancet 2016:387:649-60.

30 Millar M, Seale J, Greenland M, et al. The microbiome of infants recruited to a randomised placebo-controlled probiotic trial (PIPs trial). EBioMedicine 2017;20:255-62

31 Deshpande G, Rao S, Athalye-Jape G, et al. Probiotics in very preterm infants: the PIPS trial. The Lancet 2016;388.

32 Gotoh A, Katoh T, Sakanaka M, et al. Sharing of human milk oligosaccharides degradants within bifidobacterial communities in faecal cultures supplemented with Bifidobacterium bifidum. Sci Rep 2018;8:13958. 